\title{
Production of deoxynivalenol by Fusarium graminearum Schwabe in culture and its toxicity to wheat germlings in relation to virulence
}

\author{
Pakdaman B. S., Mohammadi Goltapeh E. *, Allameh A. A. and Alizadeh A. \\ Department of Plant Pathology, Faculty of Agriculture, Tarbiat Modarres University, Tehran, Iran.
}

Accepted 7 March, 2011

\begin{abstract}
Seven isolates of Fusarium graminearum obtained from Seed and Plant Improvement Institute were grown on autoclaved barley grains and toxicity of their cultures to wheat germinating seeds was tested using their semi-purified phytotoxins. The extracts of isolates 167, 169, 173 and 179 did not have considerable inhibitory effects on wheat seed germination, whereas that of $161(a=0.05)$ and two other isolates 131 and 164 displayed a significant inhibitory effect on germination. However, no correlation was found between virulence and toxicity of the isolates examined. Also, the effect of light was studied in relation to wheat germling level of tolerance. Six isolates were investigated for their ability of deoxynivalenol synthesis. Thus, their crude extracts were prepared and partially purified, and subjected to thin layer chromatography with chloroform (acetone and hexane) ethyl acetate solvent systems on fluorescent silica gel plates (60 meshes) using a commercial standard deoxynivalenol ( $1 \mathrm{mg} / \mathrm{ml}$, Sigma Co., USA) along with the samples. The spots of interest were detected and analyzed under ultraviolet (UV) lights (254 and $365 \mathrm{~nm}$ ). The isolate 161 produced the most amount of deoxynivalenol compared to others. Also, no correlation was found between virulence and toxigenicity of the isolates.
\end{abstract}

Key words: Phytotoxicity, fusarium head blight, head scab, virulence, deoxynivalenol, mycotoxin, phytotoxin, Fusarium graminearum.

\section{INTRODUCTION}

In many areas of the world, the important cereals (corn and wheat) are very susceptible to infection by Fusarium species, and infected seeds may be toxic and poisonous for human or animals consuming such products (Bosch et al., 1989). Four Fusarium species, Fusarium crookwellense, Fusarium culmorum, Fusarium graminearum, and Fusarium sambucinum, of section discolor produce mainly diacetoxyscirpenol and deoxynivalenol (Desjardins et al., 1993). F. graminearum produces a set of chemicals of different biogenic origins and toxicities, and various strains of the fungus produce various mixtures of metabolites such as; deoxynivalenol, 3/15-acetyldeoxynivalenol, zearalenone, culmorin, culmorone, dihydroxycalonectrin, sambucinol, sambucoin, butenolide, and fusarin C (Farber and Sanders, 1986; Greenhalgh et al., 1984, 1986). Of 40 trichothecenes identified, nivalenol and deoxynivalenol are the most predominant mycotoxins contaminating cereal grains (Ueno, 1983). Based on the production of these metabolites and their derivatives, F. graminearum strains are divided into two main chemotype groups. One primarily produces deoxynivalenol or its acetylated forms, and the other produces nivalenol and its derivatives (Miller et al., 1991). Isolates of $F$. graminearum recovered 
Table 1. Characteristics or F. graminearum isolates studied.

\begin{tabular}{cll}
\hline Isolate No. & Host wheat cultivar/line & Geographic location \\
\hline 131 & Falat & Garakheil, Gaemshahr \\
161 & Falat & Dasht-e-Naz, Sari \\
164 & Hirmand & Rostamklar, Behshahr \\
167 & Golestan & Chomadzi, Gorgan road \\
169 & Falat & Sorkhanklateh, Gorgan \\
173 & Falat & Mohammad abad, Gorgan \\
179 & Hirmand & Iraqimaalleh, Gorgan \\
\hline
\end{tabular}

from Mazandaran province are placed in the first group (Zamani-Zadeh and Khoursandi, 1990).

However, the occurrence of nivalenol producing isolates of $F$. graminearum has been also recorded in Iran (Etaati, 2002). The severity of Fusarium infection and of trichothecene contamination increases with wet weather at harvest and with storage under conditions of high relative humidity (Desjardins et al., 1993). Regardless the kind of metabolites produced, these compounds are associated with some disorders such as livestock emesis, diarrhea, decreased feed consumption, skin irritation, hemorrhage, reproductive problems and hematological changes (Abramson et al., 1993). Therefore, their occurrence in cereals is of importance in agriculture and food industries (Yao et al., 1996), and endangers social safety.

With plants, some studies carried out on the effects of trichothecenes have been related to general impressions such as wilting, chlorosis, necrosis or inhibited growth of whole plants or plant organs (Brian et al., 1961). Trichothecenes prevent cell division (Linnainmaa et al., 1979), and reduce plant tissue development, increased by growth regulator substance, clearly through indole acetic acid inhibition (Brian et al., 1961; Cole et al., 1981; Freeman, 1955; Stahl et al., 1973). The inhibitory effect of the Fusarium toxins on the in vitro germination of wheat seed and the growth of the coleoptiles has been indicated before (Miller et al., 1985; Pakdaman et al., 2003,2006 ), and therefore, there might be a possible relation between phytotoxicity of Fusarium isolates in vitro, and their virulence in planta. The main purpose of this study was to evaluate the phytotoxicity of semipurified extracts of different isolates of $F$. graminearum to investigate the possible correlation between toxin production and their virulence.

\section{MATERIALS AND METHODS}

Seven isolates of $F$. graminearum were obtained from Seed and Plant Improvement Institute, Karaj. Information about these isolates is provided in Table 1. Isolates were grown in Petri plates containing Potato Dextrose Agar (PDA) medium, incubated at $26^{\circ} \mathrm{C}$ under dark conditions for 3 days. To extract toxins, the method of extraction from whole mass of fungus and culture medium was applied.
A medium containing barley seeds saturated (wetted) with $1 \%$ sugar solution was prepared and sterilized at $121^{\circ} \mathrm{C}$ for $15 \mathrm{~min}$, then inoculated by a $5 \mathrm{~mm}^{2}$ disc of actively growing mycelium of the fungus per bottle of $100 \mathrm{~g}$ of grains and incubated in dark, at $26^{\circ} \mathrm{C}$ for 6 weeks. The seed colonized by each fungal isolate were allowed to completely dry in an oven at 60 to $70^{\circ} \mathrm{C}$ and then ground, and blended in ethanol:water (3:1) for $1 \mathrm{~h}$ on a shaker (180 to $200 \mathrm{rpm}$ ). Aliquots of $100 \mathrm{ml}$ of the filtrate were concentrated in an oven at 60 to $70^{\circ} \mathrm{C}$, and the concentrated filtrates $(50 \mathrm{ml})$ were defatted twice with normal heptan $(50 \mathrm{ml})$ on a shaker $(50 \mathrm{rpm})$ for $30 \mathrm{~min}$. The lower phase was re-extracted with chloroform (100 ml) on a shaker (180 rpm) and then left in a refrigerator overnight. The upper phase was collected, and then kept in a desiccator at $75^{\circ} \mathrm{C}$, then dissolved in ethanol:water solution (3:1) and the final volume was adjusted to $3.5 \mathrm{ml}$.

Collections were individually poured into small vials and dried completely. Then methanol $(400 \mu \mathrm{l})$ was added to each vial to redissolve the preparation. Thin layer chromatography (TLC) was performed using a solvent system containing $20 \mathrm{ml}$ of ethyl acetate:hexane (3:1). Standard deoxynivalenol sample $(1 \mathrm{mg} / \mathrm{ml})$ was chromatographed along with the samples. Amounts used were equal to $2 \mu \mathrm{l}$ per isolate extract and $5 \mu \mathrm{l}$ for control. TLC plates were of 60 meshes with a fluorescence of yellow-green colour under wave length of $254 \mathrm{~nm}$. A similar analysis was performed with $20 \mathrm{ml}$ of chloroform:acetone (2:3) solvent system and $10 \mu \mathrm{l}$ samples prepared by adding $2 \mathrm{ml}$ methanol to dried extracts and $5 \mu \mathrm{l}$ standard deoxynivalenol $(1 \mathrm{mg} / \mathrm{ml})$ blotted along with them.

Toxicity test was based on NGT $\mathrm{C} 80_{0}$ method (Buerstmayr et al., 1997) using a base medium made of water and agar ( $8 \mathrm{~g} / \mathrm{L})$ with $30,35,40,45,50$ and $100 \mathrm{ppm}$ of a mixture containing semipurified phytotoxins. The wheat cultivar used to bioassay was Attila.

After germination of $80 \%$ of seeds in controls without phytotoxic extracts, half of Petri dishes were put, exposed to sun light and others in darkness. Temperature was adjusted at $25^{\circ} \mathrm{C}$. There were three Petri dishes per treatment each containing 20 seeds. Data related to the virulence of Fusarium isolates used (Figure 1) were provided by Cereals Pathology Division of Seed and Plant Production and Improvement Institute, Karaj. These data had been obtained by the determination of the mortality percentage of a Fusarium head blight susceptible wheat cultivar grains inoculated with the spore suspensions of the isolates studied.

\section{RESULTS AND DISCUSSION}

With an eye to the importance of trichothecenes, this study was conducted to reveal their (particularly deoxynivalenol) possible role in the virulence of $F$. graminearum on wheat plants. The importance of the identification of virulence factors in a pathosystem and its application in the control of plant diseases has been 


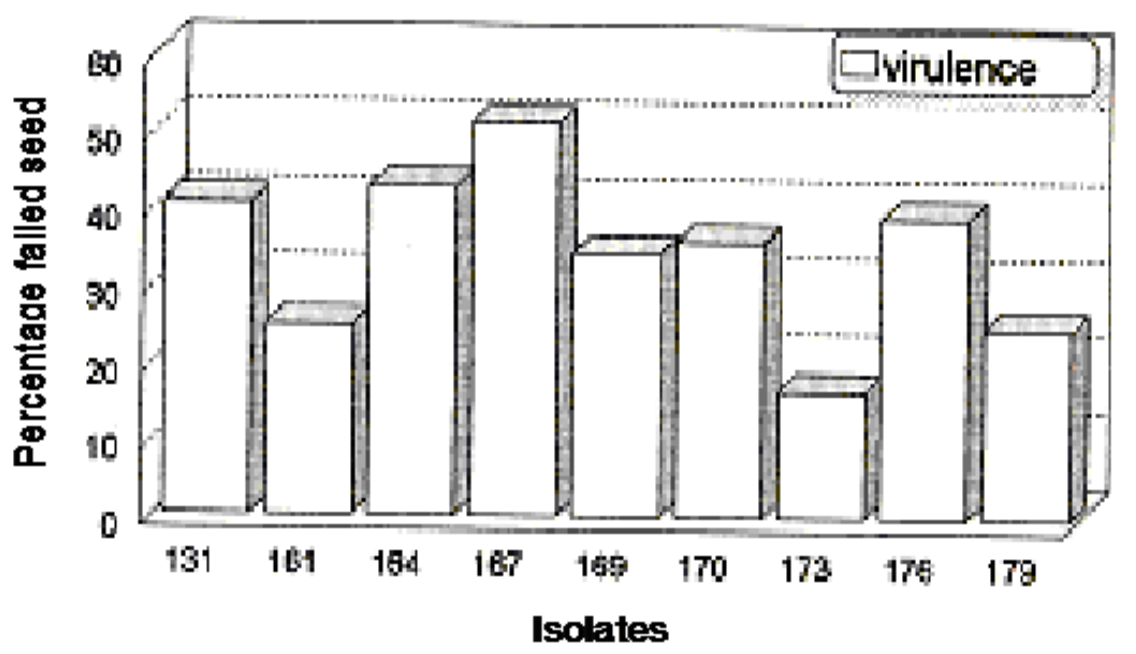

Figure 1. The relative virulence of $F$. graminearum isolates based on the percentage of the wheat seed failed to germinate (based on the data from PSSII).

previously discussed (Desjardins et al., 1993).

Based on TLC data, isolate number $161\left(\mathrm{I}_{45}\right)$ produced more quantities of deoxynivalenol in barley medium as compared to others. The fluorescent spots related to deoxynivalenol were clearly visible for isolate number 164 $\left(\mathrm{I}_{47}\right)$ and $131\left(\mathrm{I}_{42}\right)$. However, other spots related to unknown trichothecenes, were also seen on TLC plates. No attempts were made for their identification.

The fluorescent spots visible under ultraviolet (UV) radiations (254 and $365 \mathrm{~nm}$ ) were potentially related to deoxynivalenol with $\mathrm{R}_{f}$ quantities approximately equal to 23.26 and $30.08 \%$ related to the solvent systems, respectively. Other isolates examined, did not show any spots comparable to deoxynivalenol. Evidently, some isolates produce deoxynivalenol and therefore belong to the Chemotype I.

Also, other spots related to other metabolites were found on the TLC plates, however, because of the lack of the mycotoxin standards, their identification was ignored. It was also notable that the six-month-cultured isolate number 164 produced a compound that was not confronted with other young cultures of this isolate, indicating that with the elapse of time, there are new metabolites that are produced by the same isolate and reach to an extent detectable by TLC. However, the possibility of the release and accumulation of this compound from the plant-based substrate cannot be excluded, although the fungal origin of the spot seems more probable, as a similar spot is found also near to the upper edge of the plate with the isolate number 131 (Figure 2).

Phytotoxicity of various isolates was considerably different $\left(F=17.09^{* *}\right)$. Of seven isolates studied, only three namely; 131,161 and 164 were significantly toxic, and others were not toxic and behaved similar to controls (Figure 2).
Isolates 164 and 131 produced higher amounts of the toxic principal, and their semi-purified toxin extracts considerably inhibited wheat seed germination. Not only inhibitory effects of semi-purified phytotoxins caused the ratio of germinated seeds to the total number of seeds to decrease, but also the growth rates of primitive roots and coleoptile decreased due to the toxicity of fungal extracts (Figure 3). Our results showed that at least some of the isolates originating from Mazandaran province are capable of synthesizing deoxynivalenol which belong to the Chemotype I. This result is in accordance with the results obtained by Zamani-Zadeh and Khoursandi (1995). Occurrence of $F$. graminearum isolates belonging to Chemotype II has been also reported from Iran (Etaati, 2002). The production of trichothecenes such as deoxynivalenol and specially nivalenol by the isolates from Iran is of concern for human and livestock health particularly in Northern provinces, and may cause epidemics when climatic conditions are suitable for rapid growth and development of head scab disease.

A significant difference was observed among various concentrations effects of toxin $\left(F=14.33^{* *}\right)$ on coleoptile growth. Means comparative analysis by Duncan test $(\alpha=$ 0.05 ) demonstrated that the concentrations more or less than $50 \mathrm{ppm}$ not only had not caused the growth to decrease, but also they had stimulated the growth as an apparently positive factor, an effect that has been observed before (Alizadeh and Zhange, 1998) personal communication. Semi-purified toxic extract $(50 \mathrm{ppm})$ from the culture of the isolate number 164 reduced the growth of wheat coleoptile in accordance with the results obtained by Brian et al. (1961) and Wakulinski (1989).

Interactions between light and concentrations, variety and concentrations and among all the factors were highly significant $(\alpha=0.01)$. Our results are in agreement with the studies of Buerstmayr et al. (1997) and Packa and 


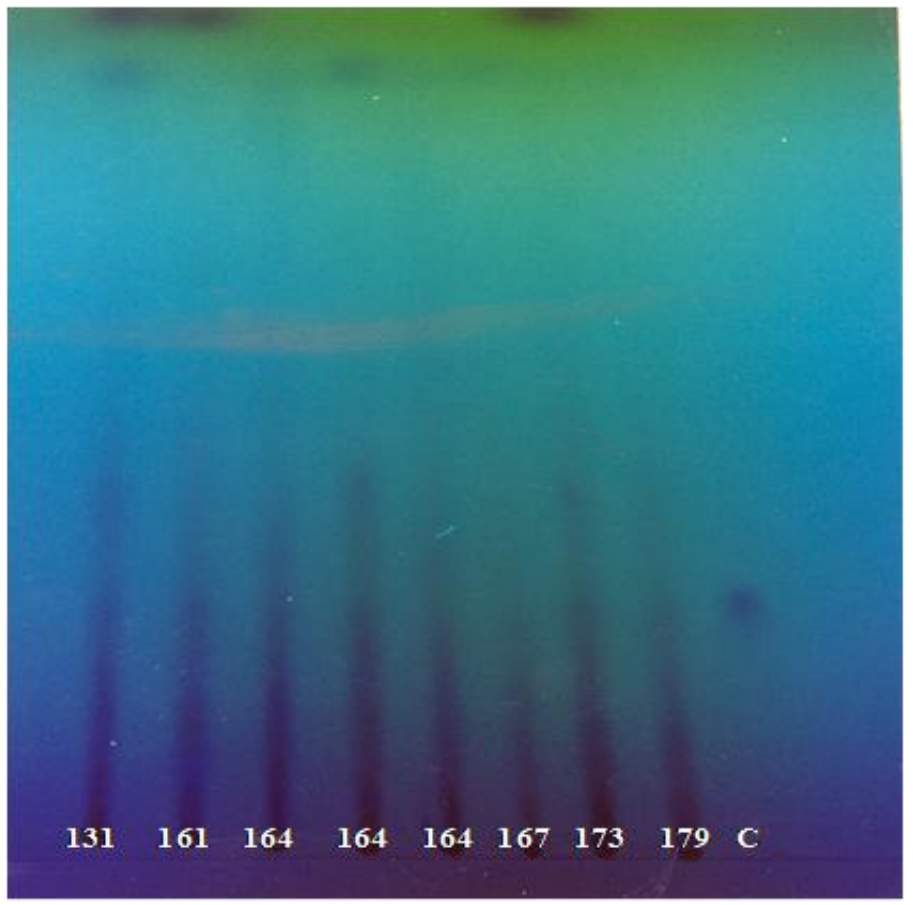

Figure 2. Thin layer cheomatography with chloroform-aceton; the isolate number 164 , from left to right: synchronously cultivated with other isolates, 6 months old, and freshly cultured.

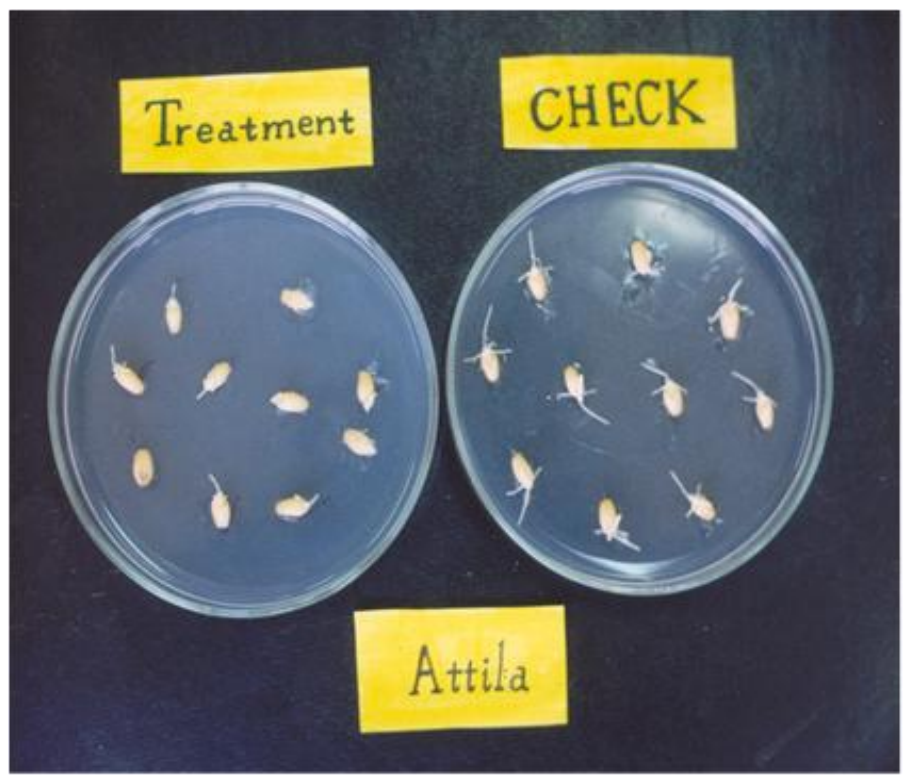

Figure 3. Effect of $F$. graminareaum phytotoxic extract (50 ppm) on seed germination in wheat susceptible cultivar Attila.

Koczowska (1987), which have demonstrated the production of toxin(s) by Fusarium in culture resulting in reduced germination and primary root and stem (coleoptile) growth (Figure 4).
Desjardins et al. (1996), Proctor et al. (1995), Eudes et al. (2001) and Dyer et al. (2005) have shown a correlation between phytotoxic trichothecenes and virulence. With our experiments, no correlation was generally found $\left(R=0.51^{\text {ns }}\right)$ 


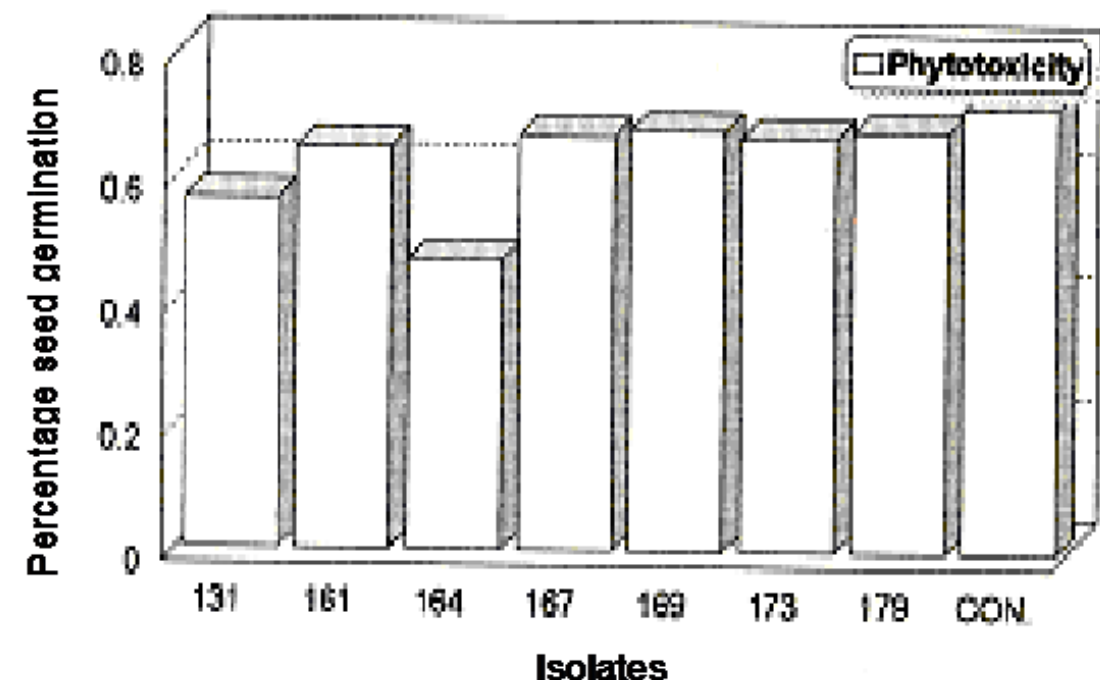

Figure 4. The relative phytotoxicity of $F$. graminearum isolates based on their semi-purified extract potentials for wheat seed germination inhibition.

between isolate phytotoxicity and virulence, so that the isolate number 161 produced the most amount of dissolved organic nitrogen (DON), but it was not so toxic overall. In contrast, the isolate number 164 with lower DON production potential caused the most phytotoxicity. It seems that the reason of this controversy is the effect of the DON concentration on its toxicity, and also the different genetic bases of different isolates. The influence of mycotoxin chemotype in determination of pathogenicity of $F$. graminearum strains on a particular host plant has been previously reported (Carter et al., 2002). Such an effect of $F$. graminearum genetic diversity of trichothecene production on the pathogen virulence has been documented with strains from Nepal (Desjardins et al., 2004). However, some other factors may involve in virulence. For example, one of the factors concerned to this result may be that any cultivar has its own potentials for tolerance of toxins; therefore, it is necessary to test both the characteristics with each given cultivar. It is also known that different isolates of $F$. graminearum have various potentials for production of mycotoxins in vitro (Miller et al., 1983; Neish and Cohen, 1981) and in the field (Thiel et al., 1982). Our results also are in accordance with that of Gang et al. (1988), and this interpretation is fortified by Miedaner et al. (1997).

Regardless of concentrations, germlings had more growth when exposed to the sun light, and this is in accordance with the results obtained by Yao et al. (1996) that the head scab resistant cultivars were capable of fungal toxin conversion to a Compound $X$ through its biodegradation, a compound which was no longer effective on wheat tissues, but able to prevent Gibberella zeae spore germination.

Abbreviations: TLC, Thin layer chromatography; DON, dissolved organic nitrogen.

\section{REFERENCES}

Abramson D, Clear RM, Smith DM (1993). Trichothecene production by Fusarium spp. isolated from Manitoba grain. Can. J. Plant Pathol. 15:147-152.

Brian AT, Dawkins AW, Grove JF, Hemming HG, Lowe D, Norris GL (1961). Phytotoxic compounds produced by F. equiseti. J. Exp. Bot. 12:1-12.

Bosch U, Mirocha CJ, Abbas HK, di Menna M (1989). Toxicity and toxin production by Fusarium isolates from New Zealand. Mycopathologia 108:73-79.

Buerstmayr H, Lemmens M, Grausgruber H, Ruckenbauer P (1997). Breeding for scab resistance in wheat: Inheritance of resistance and possibilities for in vitro selection. In: Fusarium Head Scab: Global Status and Future Prospects. Edited by H.J. Dubin, L. Gilchrist, J. Reeves, and A. McNab. D.F.: CIMMYT.

Carter JP, Rezanoor HN, Desjardins AE, Plattner RD, Nicholson P (2002). Variation in pathogenicity associated with the genetic diversity of Fusarium graminearum. Eur. J. Plant Pathol. 108:571583.

Cole RJ, Doner JW, Cox RH, Cunfer HG, Cutler HG, Stuart BP (1981). The isolation and identification of several trichothecene mycotoxins from Fusarium heterosporum. J. Nat. Prod. 44:324-330.

Desjardins AE, Jarosz AM, Plattner RD, Alexander NJ, Brown DW, Jurgenson JE (2004). Patterns of trichothecene production, genetic variability, and virulence to wheat of Fusarium graminearum from smallholder farms in Nepal. J. Agric. Food Chem. 52:6341-6346.

Desjardins AE, Proctor RH, Bai G, McCormick SP, Shaner G, Buechley G, Hohn TM (1996). Reduced virulence of trichothecene-non producing mutants of Gibberella zeae in wheat field tests. Mol. PlantMicrobe Int. 9:775-781.

Desjardins AE, Hohn TM, McCormick SP (1993). Treichothecene biosynthesis in Fusarium species: chemistry, genetics, and significance. Microbiol. Rev. 57:595-604.

Dyer RB, Plattner RD, Kenra DF, Brown DW (2005). Fusarium graminearum TRI14 is required for high virulence and DON production on wheat but not for DON sythesis in vitro. J. Agric. Food Chem. 53:9281-9287.

Etaati M (2002). Development of an accurate and sensitive bioassay method for evaluation of phytotoxins production in Fusarium 
graminearum isolates. Thesis submitted in partial fulfillment of the requirements for the degree of M. Sc. In Plant Pathology. Faculty of Agriculture, Tarbiat Modarres University. P. 148.

Eudes F, Comeau A, Rioux S, Collin J (2001). Impact of trichothecenes on Fusarium head blight [Fusarium graminearum] development in spring wheat [Triticum aestivum]. Can. J. Plant Pathol. 23:318-322.

Farber JM, Sanders GW (1986). Production of Fusarin C by Fusarium spp. J. Agric. Food Chem. 34:963-966.

Freeman GG (1955). Further biological-properties of trichothecin, an antifungal substance from trichothecium roseum link, and its derivatives. J. Genet. Microbiol. 12:213-221.

Gang G, Miedaner T, Schuhmacher U, Schollenberger M, Geiger HH (1998). Deoxynivalenol and nivalenol production by Fusarium culmorum isolates differing in aggressiveness toward winter rye. Phytopathology 88:879-884.

Greenhalgh R, Levandier D, Adams W, Miller JD, Blackwell BA, Mc Alees AJ, Taylor A (1986). Production and characterization of deoxynivalenol and other secondary metabolites of fusarium culmorum (cmi 14764, hlx 1503). J. Agric. Food Chem. 34:98-102.

Greenhalgh R, Meier Rm, Blackwell Ba, Miller JD, Taylor A, AP Simon JV (1984). Minor Metabolites of Fusarium Roseum (Atcc 28114). J. Agric. Food Chem. 32:1261-1264.

Linnainmaa K, Sorsa M, Nus T (1979). Epoxytrichothecene mycotoxins as a-mitotic agents in allium. Hereditas 90:151-156.

Miedaner T (1997). Breeding wheat and rye for resistance to Fusarium diseases. Plant Breed. 116:201-220.

Miller JD, Greenhalgb R, Wang YZ, Lu M (1991). Trichothecene chemotypes of three fusarium species. Mycologia 83:121-130.

Miller JD, Taylor A, Greenhalgh R (1983). Production of deoxynivalenol and related compounds in liquid culture by Fusarium graminearum. Can. J. Microbiol. 29:1171-1178.

Miller JD, Young JC, Sampson DR (1985). Deoxynivalenol and fusarium head blight resistance in spring cereals. Phytopathologische Zeitschrift. 113:359-367.

Neish Ga, Coben H (1981). Vomitoxin and zearalenone production by Fusarium graminearum from winter wheat and barley in Ontario. Can. J. Plant Sci. 61:811-815.

Packa D, Koczowska I (1987). The effect of Fusarium culmorum and Fusarium nivale metabolites on gennination, seedling growth and cytological disturbances of rye, wheat and triticale. Mycotoxin research, special edition. European seminar, Fusarium mycotoxins, taxonomy. Pathogenicity pp. 82-85.

Pakdaman BS, Mohammadi GE, Alizadeh A, Allameh A (2003). Application of semi-purified phytotoxins of Fusarium graminearum for evaluation of head blight resistance in wheat cultivars. J. Agric. Sci. Nat. Resour. 2:137-147.
Pakdaman BS, Mohammadi GE, Alizadeh A (2006). Effect of Fusarium graminearum phytotoxins on germinating seeds and tissue of wheat in relation to fusarium head blight resistance. Indian Phytopathol. 59:27-31.

Proctor RH, Hohn TM, Mccormick SP (1995). Reduced virulence of Gibberella zeae caused by disruption of a trichothecene toxin biosynthetic gene. Mol. Plant-microbe Int. 8:593-601.

Stahl C, Vanderhoef L, Siegel N, Helgeson JP (1973). Fusarium tricinctum $t-2$ toxin inlubits auxin-promoted elongation in soybean hypocotyl. Plant Physiol. 52:663-666.

Thiel PG, Harasas WF0, Meyer CJ (1982). Natural occurrence of Fusarium toxins in maize from Transkei. Proc. Int. IUP AC Symp. Mycotoxins, Phycotoxins, 5th. Sept. Vienna. pp. 126-129.

Ueno Y (1983). Trichothecenes- chemical, biological and toxological aspects. Tokyo: Kodansh Ltd. Tokyo and Elsevier Sc. Pub. Co., Amsterdam. P. 313.

Wakulinski W (1989). Phytotoxicity of the secondary metabolites of fungi causing wheat head fusariosis (head blight). Acta. Physiol. Plant. 11:301-306.

Yao Q, Liu Z, Zeng YQ, Liu Z, Zeng Y (1996). Detoxification of deoxynivalenol by scab resistant wheat and bioactivities of the product. Acta. Mycol. Sinica. 15:59-64.

Zamani-Zadeh HR, Khoursandi H (1995). Qccurrence of Fusarium species and their mycotoxins in wheats in Mazandaran Province. Iranian J. Plant Pathol. 31:24-37. 